\title{
Prevalence of K13-propeller polymorphisms in Plasmodium falciparum from China-Myanmar border in 2007-2012
}

Zenglei Wang ${ }^{1}$, Sony Shrestha ${ }^{1}$, Xiaolian Li', Jun Miao ${ }^{1}$, Lili Yuan ${ }^{1,2}$, Mynthia Cabrera' ${ }^{1}$ Caitlin Grube', Zhaoqing Yang ${ }^{2}$ and Liwang Cui ${ }^{1 *}$

\begin{abstract}
Background: The recent emergence and spread of artemisinin resistance in the Greater Mekong Subregion poses a great threat to malaria control and elimination. A K13-propeller gene (K13), PF3D7_1343700, has been associated lately with artemisinin resistance both in vitro and in vivo. This study aimed to investigate the $K 13$ polymorphisms in Plasmodium falciparum parasites from the China-Myanmar border area where artemisinin use has the longest history.

Methods: A total of 180 archived P. falciparum isolates containing 191 parasite clones, mainly collected in 2007-2012 from the China-Myanmar area, were used to obtain the full-length K13 gene sequences.

Results: Seventeen point mutations were identified in $46.1 \%$ (88/191) parasite clones, of which seven were new. The F446I mutation predominated in $27.2 \%$ of the parasite clones. The C580Y mutation that is correlated with artemisinin resistance was detected at a low frequency of $1.6 \%$. Collectively, $43.1 \%$ of the parasite clones contained point mutations in the kelch domain of the $K 13$ gene. Moreover, there was a trend of increase in the frequency of parasites carrying kelch domain mutations through the years of sample collection. In addition, a microsatellite variation in the N-terminus of the K13 protein was found to have reached a high frequency (69.1\%).

Conclusions: This study documented the presence of mutations in the K13 gene in parasite populations from the China-Myanmar border. Mutations present in the kelch domain have become prevalent $(>40 \%)$. A predominant mutation $\mathrm{F} 446 \mathrm{I}$ and a prevalent microsatellite variation in the $\mathrm{N}$-terminus were identified, but their importance in artemisinin resistance remains to be elucidated.
\end{abstract}

Keywords: Malaria, Plasmodium falciparum, artemisinin resistance, K13 gene, F3D7_1343700

\section{Background}

Malaria has been scourging human beings for millennia, and remains responsible for over 430,000 child deaths in Africa every year [1]. The world has made remarkable strides in battling against this ancient enemy during the past decade, reducing by an impressive $47 \%$ in mortality rate globally between 2000 and 2013 [1]. However, parasite resistance to anti-malarials remains an ever-present obstacle to eliminate malaria. Chloroquine and sulphadoxinepyrimethamine have failed as crucial medicines in the treatment of the deadly malaria parasite Plasmodium

\footnotetext{
* Correspondence: luc2@psu.edu

'Department of Entomology, The Pennsylvania State University, University Park, PA 16802, USA

Full list of author information is available at the end of the article
}

falciparum due to the emergence and rapid spread of drug resistance. More worryingly, resistance to artemisinin (ART) family drugs has been detected and is spreading in Southeast Asia [2-4], posing a major threat to the implementation of artemisinin-based combination therapy (ACT) as a defensive line against $P$. falciparum.

ART resistance is manifested clinically as delayed parasite clearance half-life ( $>5$ hours) in vivo [4,5]. An in vitro ring-stage survival assay $\left(\mathrm{RSA}_{0-3 \mathrm{~h}}\right)$, which measures the percentage of early ring-stage parasites $(0-3$ hrs postinvasion of red blood cells (RBCs)) that survive exposure to a pharmacologically relevant concentration of dihydroartemisinin, has been developed to reflect this ART resistance phenotype [6]. Recent work has associated ART resistance with mutations in the propeller domain of a 
kelch gene on chromosome 13 (PF3D7_1343700, K13 gene) [5]. The K13 mutation M476I was initially identified in a Tanzanian P. falciparum strain that had undergone in vitro ART selection for five years. Research on parasite isolates from Cambodia, where ART resistance was first observed, identified K13 mutations Y493H, R539T and C580Y to be associated with delayed clearance [5]. These mutations were confirmed to contribute to in vitro ART resistance through genetic manipulations of the K13 gene $[7,8]$. A large, multicentre, clinical study further indicates that ART resistance is spreading in the Greater Mekong Subregion (GMS), where single-point mutations in the propeller domain of $K 13$ after the position 440 are collectively associated with ART resistance [4]. Surveys conducted in different regions showed that $K 13$ mutations associated with ART resistance were restricted to certain areas of the GMS, including Cambodia, Thailand, Myanmar, and Vietnam. The C580Y mutation is the predominant one approaching fixation in Western Cambodia [5,9-11]. These mutations have not been detected in Bangladesh and Laos $[4,10,12]$. Surveys of African parasite populations, while having found a diverse array of mutations within the $K 13$ gene, did not detect those mutations associated with ART resistance [13-17].

ART family drugs have been used in China's Yunnan Province since the late 1970s [18]. In recent years, clinical efficacy studies conducted in this region showed that artemisinin drugs for treating falciparum malaria remain highly effective $[19,20]$. However, the proportion of day 3 parasite-positive cases in one study reached 18.5\% [20], well above the $10 \%$ threshold set by the World Health Organization as a proxy indicator of suspected ART resistance [21], suggesting possible emergence of ART resistance in this area. In this study, the polymorphisms of $K 13$ genes in parasite populations along the China-Myanmar border were investigated, and the presence of $K 13 \mathrm{mu}-$ tations that are associated with clinical ART resistance in this region was demonstrated using longitudinally archived parasite samples. More importantly, parasite strains carrying wild-type $K 13$ alleles have been declining through the six years of sample collection.

\section{Methods}

Collection of parasite clinical isolates, DNA extraction and genotyping

Plasmodium falciparum clinical isolates were collected during the period 2004-2012 in malaria clinics located along the China-Myanmar border, cultured and archived [22]. A total of 180 samples were analysed including two collected in 2004, 25 in 2007, 47 in 2008, 78 in 2009, 11 in 2010, five in 2011, and 12 in 2012. Genomic DNA was extracted using the Wizard ${ }^{\circ}$ Genomic DNA Purification Kit (Promega, WI, USA). Parasite samples were genotyped at msp1, msp 2 and glurp using previously described methods [23-25] to distinguish single from mixed-strain infections [26].

K13 propeller gene amplification and sequencing analysis The full-length $K 13$ gene was amplified by high-fidelity PCR using Advantage HD DNA Polymerase Mix (Clontech, Mountain View, CA, USA) and primers KP13-F (5' -TATAACAAGGCGTAAATATTCGTG-3') and KP13-R (5' -TGTGCATGAAAATAAATATTAAAG AAG-3'). PCR reactions were performed in $37.5 \mu \mathrm{l}$ with $0.5 \mu \mathrm{M}$ of DNA template, $0.2 \mu \mathrm{M}$ of each primer, $3.75 \mu \mathrm{l}$ of $10 \times$ PCR buffer, 3.75 units of DNA polymerase mix, and $0.2 \mathrm{mM}$ of dNTP mix. Reaction conditions consisted of an initial denaturation at $95^{\circ} \mathrm{C}$ for $5 \mathrm{~min}$ followed by 35 cycles of $95^{\circ} \mathrm{C}$ for $30 \mathrm{sec}, 55^{\circ} \mathrm{C}$ for $30 \mathrm{sec}$ and $68^{\circ} \mathrm{C}$ for $3 \mathrm{~min}$, and a final extension step for $7 \mathrm{~min}$ at $68^{\circ} \mathrm{C}$. PCR products were sequenced in both directions using sequencing primers KP13-65 F (5'-GGGAATCT GGTGGTAACAGC-3'), KP13-640R (5'-CACTAGCAT CACTTAATTCCGTT-3'), KP13-517 F (5'-GATGCAG CAAATCTTATAAATGATG-3'), KP13-759 F (5' -GGAA AGAGTACGATTGTACAAAG-3'), KP13-1363R (5'-CT ACACCATCAAATCCACCTATA-3'), KP13-1595 F (5' GTGGTGTTACGTCAAATGGTAG-3'), and KP13-R. Sequences were assembled by DNASTAR (WI, USA) with manual editing. Alignment of DNA sequences were performed using MEGA 6.0 [27] with the $K 13$ sequence of the 3D7 clone (PF3D7_1343700) retrieved from PlasmoDB as the reference. To superimpose the mutations in the $K 13$ protein, the $3 \mathrm{D}$ structure of the $K 13$ protein was predicted by Phre2 online protein structure prediction tool [28]. The chosen template retained 100\% confidence based on homology assessment and model prediction quality.

\section{Statistical analysis}

Fisher's exact test was done to assess difference in the frequency of mutations between years using GraphPad Prism 5 (GraphPad Software, Inc. La Jolla, CA, USA).

\section{Results}

The full-length K13-propeller genes were sequenced from a total of 180 clinical isolates collected from malaria patients along the China-Myanmar border. Of these isolates, 178 were collected during 2007-2012, while two were collected in 2004. Genotyping of these parasites at three polymorphic genes confirmed that 169 were monoclonal infections, whereas 11 were mixed infections at one of the three loci [29]. Since each of the mixed infections contained only two allelic types, they were considered as having two parasite strains in each sample. Based on their clear sequencing chromatograms, these mixed infections were also included in the analysis. Accordingly, the 
frequencies of mutations were estimated by using 191 parasite clones in the study population.

Sequencing of the $K 13$ genes in the 191 clones revealed that 88 (46.1\%) contained single nucleotide polymorphisms (SNPs) at 17 locations. All 17 SNPs were nonsynonymous; no synonymous mutation was detected in these isolates. In addition, variations in the number of a microsatellite repeat (ATA) corresponding to amino acid positions 137-142 of K13 in 3D7 parasite were also observed. This microsatellite sequence encodes the Asn (N) residue and is present in 30.4, 69.1 and $0.5 \%$ of the 191 clones as six (wild type), eight and nine Ns, respectively. For the point mutations, ten (K189T, E252Q, R255K, P441L, F446I, N458Y, P574L, C580Y, A676D and H719N) were described previously $[4,5,30,31]$ and seven (N11Y, I352T, I376V, P443S, C469Y, L492S and F495L) were new to parasites in this region (Table 1, Figure 1). All of these mutations occurred singly, except that a double-mutation P574L and F446I was detected in one parasite clone. One major distinction of this parasite population is the predominant status of the F446I substitution, reaching a frequency of $27.2 \%$. Coincidently, this point mutation was only observed in parasites with the $8 \mathrm{~N}$ repeats at the microsatellite locus. The P574L mutation was the second most prevalent (6.7\%). The rest of mutations were all rare and occurred in one to three clones $(0.5-1.6 \%)$. It is

\section{Table 1 Amino acid substitutions in the $K 13$ gene in} parasites from the China-Myanmar border $(n=191)$

\begin{tabular}{|c|c|}
\hline Mutation & No (\%) \\
\hline $\mathrm{N} 11 \mathrm{Y}^{*}$ & $1(0.5)$ \\
\hline K189T & $3(1.6)$ \\
\hline E252Q & $1(0.5)$ \\
\hline $\mathrm{R} 255 \mathrm{~K}$ & $1(0.5)$ \\
\hline $1352 \mathbf{T}^{*}$ & $1(0.5)$ \\
\hline $1376 \mathbf{V}^{*}$ & $2(1.0)$ \\
\hline P441L & $1(0.5)$ \\
\hline P443S* & $1(0.5)$ \\
\hline F446I & $52(27.2)$ \\
\hline N458Y & $1(0.5)$ \\
\hline$C 469 Y^{*}$ & $2(1.0)$ \\
\hline L492S* & $1(0.5)$ \\
\hline$F 495 L^{*}$ & $2(1.0)$ \\
\hline P574L & $12(6.3)$ \\
\hline C580Y & $3(1.6)$ \\
\hline A676D & $2(1.0)$ \\
\hline $\mathrm{H} 719 \mathbf{N}$ & $2(1.0)$ \\
\hline In kelch domain & $79(41.3)$ \\
\hline Total & $88(46.1)$ \\
\hline
\end{tabular}

*indicates unique mutations identified in this parasite population. Letters in bold indicate mutated amino acids.

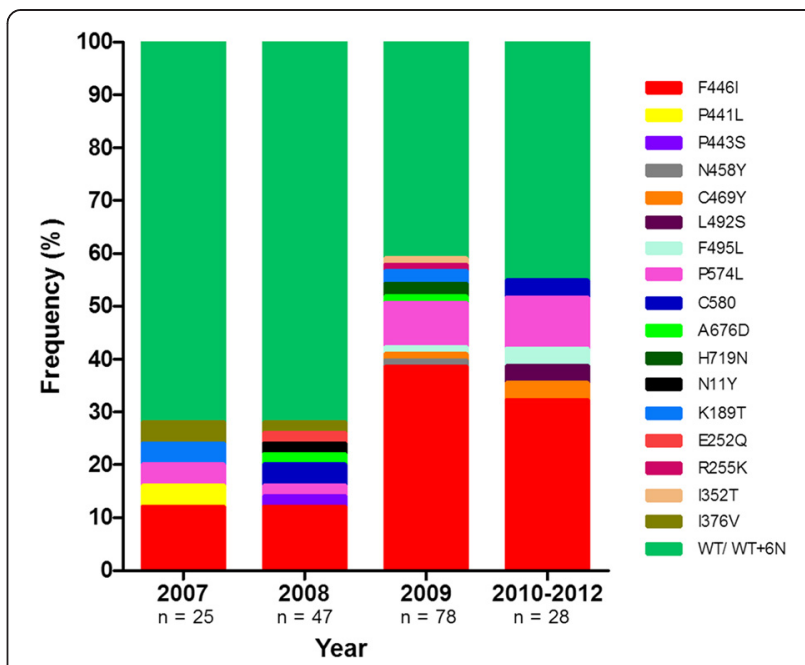

Figure 1 Prevalence of K13 mutations in parasites collected from the China-Myanmar border area in 2007-2012.

noteworthy that the C580Y mutation, which was correlated with ART resistance and prevailed in other areas of Southeast Asia, was present in only three clones (1.6\%). None of the I543T, R539T and Y493H mutations associated with delayed parasite clearance in the Cambodian isolates, or the M476I substitution selected in vitro in a Tanzanian strain, was observed in this parasite population.

The K13 protein contains a piece of Plasmodium-specific sequence and a BTB/POZ domain in the $\mathrm{N}$-terminus, and a 6-blade propeller domain in the $\mathrm{C}$-terminus (codons 441-725) [5]. Of the 17 mutations identified in this parasite population, six were distributed in the $\mathrm{N}$-terminus in nine clones, whereas the remaining 11 occurred within the kelch propeller domain in $41.3 \%$ parasite clones. Superimposing the mutations to the predicted kelch domain structure showed that five (P441L, P443S, F446I, N458Y, C469Y) were clustered in blade I, two (L492S and F495L) in blade II, two (P574L and C580Y) in blade IV, and two (A676D and H719N) in blade VI (Figure 2). A recent multicentre clinical investigation showed that various mutations occurring in the kelch domain after position 440 are collectively associated with an increase in parasite clearance half-life [4]. When the samples were stratified by the year of collection, the frequencies of all mutations in the kelch domain, as well as all mutations in K13 gene, increased during the time 2007-2012 (Figure 3). In particular, a significant increase was observed in the frequencies of mutations in the kelch domain between 2008 and 2009 ( $P<0.0001)$. However, the frequencies of mutations in the N-terminus of K13 protein decreased over the years of collection (Figure 3).

\section{Discussion}

The involvement of $K 13$ mutations in ART resistance in Cambodia encouraged molecular surveillance of $\mathrm{K} 13$ 


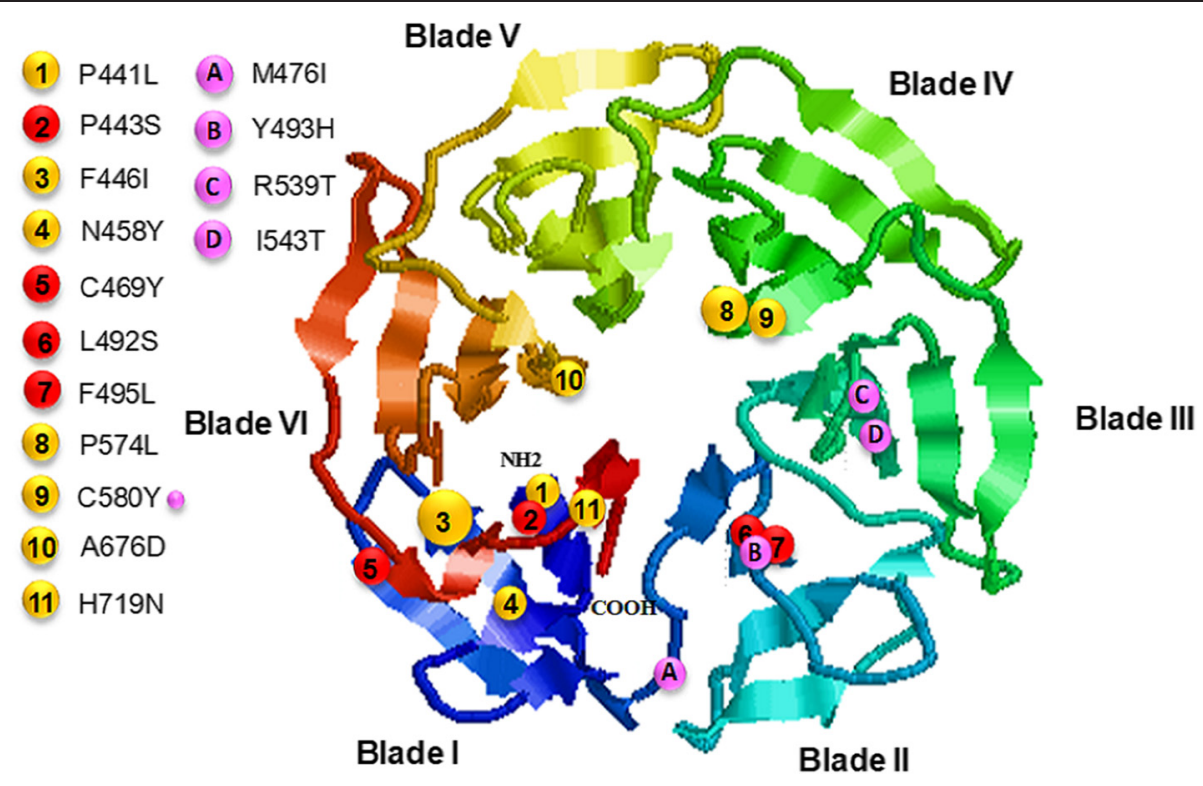

Figure 2 Distribution of the mutations in the predicted 3D model of the K13 propeller domain. The predicted structure of the propeller domain forms six propeller blades that contain predominantly strands. The locations of the various mutations are indicated by spheres, where red colour represents new mutations, orange for previous reported mutations, and pink for mutations correlated with ART resistance reported by Ariey et al. The relative frequencies of the mutations are reflected by the size of the spheres.

genes in many malaria-endemic regions. K13 mutations associated with ART resistance were mainly detected in areas of the GMS [5,9-11], with C580Y being the predominant. In contrast, surveys in Africa identified numerous mutations in the $K 13$ gene, but most occurred at low frequencies. Moreover, mutations associated with ART resistance were not observed [13-16]. In this study, sequencing of $K 13$ gene from 180 longitudinally collected parasite samples (containing 191 parasite clones) from the China-Myanmar border area identified 17 mutations (ten previously described and seven new). Compared with other surveys in Myanmar or the China-Myanmar border,

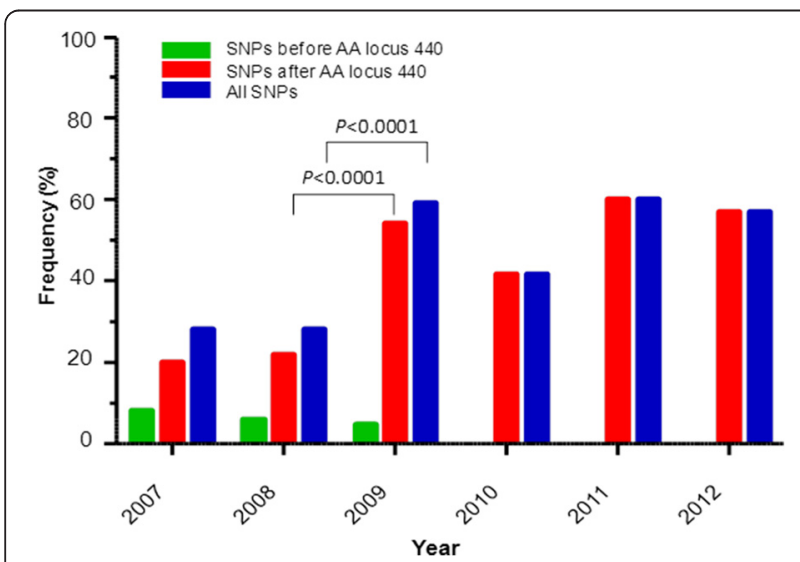

Figure 3 Frequencies of K13 mutations in 2007 to 2012. Significant increase in frequencies of mutations in the kelch domain in 2009 was noted. parasites from this study shared three mutations (F446I, P574L and A676D) with those reported by Feng et al. [30], three (P441L, N458Y and C580Y) with those reported by Nyunt et al. [9] and four (F446I, P574L, C580Yand A676D) with those reported by Tun et al. [31]. Of particular interest is the identification of the F446I as the predominant mutation in the study samples, which has reached a frequency of $27.1 \%$, coincident with results from other study (19.2\%) in this region [30]. Although other mutations were at low frequencies, they collectively gave a total of $41.3 \%$ parasites carrying mutations in the kelch domain. Among them, the predominant C580Y mutation in other regions of Southeast Asia $[4,5,10]$ was detected in $1.6 \%$ of these samples. This mutation was found to be significantly associated with prolonged parasite clearance half-life in parasites from Cambodia [5] and was confirmed to confer ART resistance by genetic manipulations $[7,8]$. Most of the mutations in the kelch domain, including the most prevalent F446I mutation, remain to be genetically characterized to determine whether they confer ART resistance.

Another intriguing phenomenon discovered in this study is the highly prevalent microsatellite variations in the N-terminus of the $K 13$ gene. Compared to the wildtype parasite, which has six $\mathrm{N}$ residues, $69.1 \%$ parasites harboured eight $\mathrm{N}$ residues. The eight $\mathrm{N}$ variation was only reported in Senegalese isolates with a frequency of 6.3\% [16]. More interestingly, the predominant mutation F446I was observed only in parasites with eight $\mathrm{N}$ repeats. In an earlier study, microsatellite variations in the 
nhe1 gene were associated with altered sensitivities to quinine in parasite population from the same area [26]. Therefore, it would be interesting to find out whether the $K 13$ microsatellite variations affect parasites' sensitivities to ART drugs.

ARTs have been used in the China-Myanmar border area for over three decades, mostly as monotherapy prior to 2005. The ACT drug deployed here is the dihydroartemisinin/piperaquine combination, compared to artesunate/mefloquine in most other areas of the GMS. One can hypothesize that it is likely that the distinct K13 mutations observed in parasites from this study site might have emerged separately as a result of selection from a different ART regimen. In addition, given the predominant status of the C580Y mutation in other parts of the GMS, it remains an open question as to whether the presence of this mutation in these samples was due to independent emergence or had spread from other areas. Furthermore, clinical efficacy studies also detected day-3 parasite positivity rate to $>10 \%$ after artesunate treatment of falciparum malaria in this area [32]. Taken together, increased surveillance and population studies are needed to determine further spread of ART resistance in the GMS.

\section{Conclusions}

Seventeen point mutations were identified in the $K 13$ gene from 180 archived parasite samples collected from the China-Myanmar border area. Analysis of the 191 parasite strains identified $43.1 \%$ carrying point mutations in the kelch domain, of which the F446I mutation reached a predominance of $27.1 \%$. Given the presence of $K 13$ mutations conferring ART resistance and detection of day- 3 parasite positivity after ART drug treatment, increased surveillance of $P$. falciparum for anti-malarial drug resistance is highly demanded.

\section{Competing interests}

The authors declare that they have no competing interests.

\section{Authors' contributions}

$Z W, X L, J M, L Y, M C, C G$, and $Z Y$ carried out the experimental work. ZW and SS participated in data analysis. ZW wrote the manuscript. ZW and LC conceived the study and participated in the design of the study. All authors read and approved the final manuscript.

\section{Acknowledgements}

This work was supported by grant from the National Institute of Allergy and Infectious Diseases, National Institutes of Health (U19 Al089672).

\section{Author details}

'Department of Entomology, The Pennsylvania State University, University Park, PA 16802, USA. '2Department of Pathogen Biology and Immunology, Kunming Medical University, Kunming, Yunnan Province 650500, China.

Received: 23 January 2015 Accepted: 16 March 2015

Published online: 18 April 2015

\section{References}

1. WHO. World malaria report 2014. Geneva: World Health Organization; 2014. http://www.who.int/malaria/publications/world_malaria_report_2014/en/ 2014.
2. Noedl H, Se Y, Schaecher $K$, Smith BL, Socheat D, Fukuda MM, et al. Evidence of artemisinin-resistant malaria in Western Cambodia. N Engl J Med. 2008;359:2619-20.

3. Dondorp AM, Nosten F, Yi P, Das D, Phyo AP, Tarning J, et al. Artemisinin resistance in Plasmodium falciparum malaria. N Engl J Med. 2009;361:455-67.

4. Ashley EA, Dhorda M, Fairhurst RM, Amaratunga C, Lim P, Suon S, et al. Spread of Artemisinin resistance in Plasmodium falciparum malaria. N Engl J Med. 2014;371:411-23.

5. Ariey F, Witkowski B, Amaratunga C, Beghain J, Langlois AC, Khim N, et al. A molecular marker of artemisinin-resistant Plasmodium falciparum malaria. Nature. 2014;505:50-5.

6. Witkowski B, Amaratunga C, Khim N, Sreng S, Chim P, Kim S, et al. Novel phenotypic assays for the detection of artemisinin-resistant Plasmodium falciparum malaria in Cambodia: in-vitro and ex-vivo drug-response studies. Lancet Infect Dis. 2013;13:1043-9.

7. Straimer JGN, Witkowski B, Amaratunga C, Duru V, Ramadani AP, Dacheux $M$, et al. K13-propeller mutations confer artemisinin resistance in Plasmodium falciparum clinical isolates. Science. 2015;347:428-31.

8. Ghorbal M, Gorman M, Macpherson CR, Martins RM, Scherf A, Lopez-Rubio JJ. Genome editing in the human malaria parasite Plasmodium falciparum using the CRISPR-Cas9 system. Nat Biotechnol. 2014;32:819-21.

9. Nyunt MH, Hlaing T, Oo HW, Tin-Oo LK, Phway HP, Wang B, et al. Molecular assessment of artemisinin-resistance markers, polymorphisms in the K13 propeller and a multidrug-resistance gene, in eastern and western border areas of Myanmar. Clin Infect Dis. 2014, doi:10.1093/cid/ciu1160.

10. Takala-Harrison S, Jacob CG, Arze C, Cummings MP, Silva JC, Dondorp AM, et al. Independent emergence of artemisinin resistance mutations among Plasmodium falciparum in Southeast Asia. J Infect Dis. 2014, doi:10.1093/infdis/jiu491.

11. Thriemer K, Hong N, Rosanas-Urgell A, Phuc BQ, Ha-do M, Pockele E. Delayed parasite clearance after treatment with dihydroartemisinin-piperaquine in Plasmodium falciparum malaria patients in Central Vietnam. Antimicrob Agents Chemother. 2014;58(12):7049-55.

12. Mohon A, Alam MS, Bayih AG, Folefoc A, Shahinas D, Haque R. Mutations in Plasmodium falciparum K13 propeller gene from Bangladesh (2009-2013). Malar J. 2014;13:431. doi:10.1186/1475-2875-13-431

13. Conrad MD, Bigira V, Kapisi J, Muhindo M, Kamya MR, Havlir DV, et al. Polymorphisms in $\mathrm{K} 13$ and falcipain-2 associated with artemisinin resistance are not prevalent in Plasmodium falciparum isolated from Ugandan children. Plos One. 2014;9:e105690.

14. Taylor SM, Parobeck CM, DeConti DK, Kayentao K, Coulibaly SO, Greenwood $\mathrm{BM}$, et al. Absence of putative artemisinin resistance mutations among Plasmodium falciparum in sub-Saharan Africa: a molecular epidemiologic study. J Infect Dis. 2014;211:680-8. doi: 10.1093/infdis/jiu467.

15. Kamau E, Campino S, Amenga-Etego L, Drury E, Ishengoma D, Johnson K, et al. K13-propeller polymorphisms in Plasmodium falciparum parasites from sub-Saharan Africa. J Infect Dis. 2014, doi: 10.1093/infdis/jiu608.

16. Torrentino-Madamet M, Fall B, Benoit N, Camara C, Amalvict R, Fall M. Limited polymorphisms in $\mathrm{k} 13$ gene in Plasmodium falciparum isolates from Dakar, Senegal in 2012-2013. Malar J. 2014;13:472. doi:410.1186/1475-2875-1113-1472.

17. Isozumi R, Uemura H, Kimata I, Ichinose Y, Logedi J, Omar AH, et al. Novel mutations in K13 propeller gene of artemisinin-resistant Plasmodium falciparum. Emerg Infect Dis. 2015;21:40-2. doi:10.3201/eid2103.140898.

18. Cui L, Su XZ. Discovery, mechanisms of action and combination therapy of artemisinin. Expert Rev Anti Infect Ther. 2009;7:999-1013.

19. Sun X, Zhang Z, Wang J, Deng Y, Yang Y, Lasi J, et al. Therapeutic efficacy and safety of compound dihydroartemisinin/piperaquine for uncomplicated Plasmodium falciparum infection in Laiza city of Myanmar bordering on China. Chin J Parasitol Parasit Dis. 2011;29:372-5.

20. Huang F, Tang L, Yang H, Zhou S, Sun X, Liu H. Therapeutic efficacy of artesunate in the treatment of uncomplicated Plasmodium falciparum malaria and anti-malarial, drug-resistance marker polymorphisms in populations near the China-Myanmar border. Malar J. 2012;11:278.

21. WHO. Global plan for artemisinin resistance containment (GPARC). 2011. http://who.int/malaria/publications/atoz/9789241500838/en/.

22. Wang Z, Parker D, Meng H, Wu L, Li J, Zhao Z, et al. In vitro sensitivity of Plasmodium falciparum from China-Myanmar border area to major ACT drugs and polymorphisms in potential target genes. PLoS One. 2012;7:e30927.

23. Kaneko O, Kimura M, Kawamoto F, Ferreira MU, Tanabe K. Plasmodium falciparum: allelic variation in the merozoite surface protein 1 gene in wild isolates from southern Vietnam. Exp Parasitol. 1997;86:45-57. 
24. Snounou G, Zhu X, Siripoon N, Jarra W, Thaithong S, Brown KN, et al. Biased distribution of msp1 and msp2 allelic variants in Plasmodium falciparum populations in Thailand. Trans R Soc Trop Med Hyg. 1999;93:369-74.

25. Roper C, Richardson W, Elhassan IM, Giha H, Hviid L, Satti GM, et al. Seasonal changes in the Plasmodium falciparum population in individuals and their relationship to clinical malaria: a longitudinal study in a Sudanese village. Parasitology. 1998;116:501-10.

26. Meng $H$, Zhang R, Yang H, Fan Q, Su X, Miao J, et al. In vitro sensitivity of Plasmodium falciparum clinical isolates from the China-Myanmar border area to quinine and association with polymorphism in the $\mathrm{Na}+/ \mathrm{H}+$ exchanger. Antimicrob Agents Chemother. 2010;54:4306-13.

27. Tamura K, Stecher G, Peterson D, Filipski A, Kumar S. MEGA6: molecular evolutionary genetics analysis version 6.0. Mol Biol Evol. 2013;30:2725-9.

28. Kelley LA, Sternberg MJE. Protein structure prediction on the Web: a case study using the Phyre server. Nat Protoc. 2009;4:363-71.

29. Yuan LL, Zhao H, Wu LO, Li XM, Parker D, Xu SH, et al. Plasmodium falciparum populations from northeastern Myanmar display high levels of genetic diversity at multiple antigenic loci. Acta Trop. 2013;125:53-9.

30. Feng J, Zhou D, Lin Y, Xiao H, Yan H, Xia Z. Amplification of pfmdr1, pfert, pvmdr1 and K13-propeller polymorphism associated with Plasmodium falciparum and Plasmodium vivax at the China-Myanmar border. Antimicrob Agents Chemother 2015, doi: 10.1128/AAC.04843-14

31. Tun KM, Imwong M, Lwin KM, Win AA, Hlaing TM, Hlaing T, et al. Spread of artemisinin-resistant Plasmodium falciparum in Myanmar: a cross-sectional survey of the K13 molecular marker. Lancet Infect Dis. 2015, doi:10.1016/ S1473-3099(15)70032-0

32. Huang F, Tang L, Yang H, Zhou S, Liu H, Li J, et al. Molecular epidemiology of drug resistance markers of Plasmodium falciparum in Yunnan Province, China. Malar J. 2012;11:243.

\section{Submit your next manuscript to BioMed Central and take full advantage of:}

- Convenient online submission

- Thorough peer review

- No space constraints or color figure charges

- Immediate publication on acceptance

- Inclusion in PubMed, CAS, Scopus and Google Scholar

- Research which is freely available for redistribution 